\title{
Le développement en question en Polynésie
} française

\section{Christian Ghasarian, Tamatoa Bambridge et Philippe Geslin}

\section{(2) OpenEdition \\ 12 Journals}

Édition électronique

URL : http://journals.openedition.org/jso/221

DOI : $10.4000 /$ jso.221

ISSN : 1760-7256

\section{Éditeur}

Société des océanistes

\section{Édition imprimée}

Date de publication : 1 décembre 2004

Pagination : 211-222

ISSN : 0300-953x

\section{Référence électronique}

Christian Ghasarian, Tamatoa Bambridge et Philippe Geslin, « Le développement en question en

Polynésie française », Journal de la Société des Océanistes [En ligne], 119 | Année 2004-2, mis en ligne le 01 décembre 2007, consulté le 19 avril 2019. URL : http://journals.openedition.org/jso/221 ; DOI : $10.4000 /$ jso. 221

() Tous droits réservés 


\section{Le développement en question en Polynésie française}

par

Christian GHASARIAN*, Tamatoa BAMBRIDGE ** et Philippe GESLIN ***

\section{RÉSUMÉ}

Le cas de la Polynésie française est tout particulièrement approprié pour appréhender par le biais du regard anthropologique les conceptions divergentes du développement. Un dilemme souvent rencontré par les responsables locaux, notamment dans les îles, est en effet de concilier le développement au sens occidental avec la logique collective très marquée en Polynésie. Or, un véritable développement ne peut pas faire l'économie de penser les concepts utilisés. Cet article pointe la dimension culturelle en jeu dans les questions de développement en Polynésie française, notamment les conceptions mòhi du temps, de l' espace, de la nature et de la collectivité, et propose quelques pistes de réflexion sur leur nécessaire prise en compte dans les politiques culturelles du développement local.

Mots-CLÉs : Rapa, développement, rationalités économiques, profit privé, logiques collectives.

\begin{abstract}
The case of French Polynesia is particularly adapted to apprehend through an anthropological approach the divergent conceptions of development. A dilemma often met by the local persons in charge, in particular in the islands, is indeed to reconcile the western ideas of development with the collective logic very important in Polynesia. However, a true development cannot avoid to think the concepts used. This article points cultural dimension underlying the issue of development in French Polynesia, in particular the mòni ma'ohri views of time, space, nature and the community. It also offers some directions of reflexionreflexion to take into account when deciding the cultural policies of the local development.
\end{abstract}

KEYwords: Rapa, development, economic rationnalities, private profit, collective logics.

« non-développement » pour s'engager dans la voie du « développement ». Des formules diverses qualifient les mêmes idées, comme celles de « modernité », de " progrès », d' " industrialisation » et, plus récemment, de « nouvelle gouvernance ». Le terme « développement» est par ailleurs aujourd'hui amendé ou amadoué - pour reprendre les termes récents d'Edgard Morin
La notion de « développement » a principalement été élaborée par les sociétés occidentales pour catégoriser les " besoins », " retards » et " difficultés » de sociétés non occidentales. Animés par des modèles de société spécifiques, les discours politiques, économiques et analytiques en la matière prennent la plupart du temps comme allant de soi la nécessité de sortir du

* Ethnologue à l'Institut d'ethnologie de Neuchâtel (Suisse), christian.ghasarian@unine.ch; ** sociologue au CNRS, tbambridge@yahoo.fr ; *** ethnologue à l'INRA, philippe.geslin@unine.ch. 
(2003) - en «développement soutenable», « humain » ou « durable », ce dernier, si l'on s'y attarde un peu, ne stigmatisant plus uniquement des pays qui dans les instances internationales passèrent des statuts de "sous-développés " à ceux de "pays en voie de développement» (Baré, 1995, 149), voire de "pays en voie de développement industriel» (PVDI) (Wisner, 1997). L'objectif politique du concept de « développement durable » est de signifier à ceux qui l'auraient oublié, que ce " processus » est affaire de tous et concerne par conséquent le monde entier.

Issues du projet des Lumières, les conceptions occidentales du développement prônent le progrès dans ses formes éducatives, technologiques et scientifiques, celui-ci devant notamment conduire à l'émancipation de l'individu, à son contrôle de l'environnement naturel et à des formes sociales de plus en plus justes et sécurisantes. Les institutions du développement mettent ainsi l'emphase idéologique sur l'accumulation des connaissances, une meilleure alimentation, une meilleure santé pour un nombre croissant de personnes, etc. L'idéologie du progrès est soutenue par l'État mais aussi par une économie de marché qui, par son système de coûts et profits, fonde la réussite des uns sur la "moindre réussite » (pour ne pas dire l'échec) des autres. L'individualisation des droits de propriété privée est idéologiquement prôné comme une condition du développement puisqu'elle est censée procurer une plus grande sécurité juridique aux populations comme aux investisseurs. Les analyses marxistes, bien que foncièrement orientées vers la modernité et la valorisation du progrès, ont depuis longtemps pointé les travers du développement généralisé de la consommation (de masse) qui y sont associés. Le modèle du développement occidental, toujours idéologiquement associé à une meilleure justice sociale, demeure cependant sous-jacent aux processus de mondialisation/globalisation actuels. Or, les conditions historiques, philosophiques et culturelles du développement au sens occidental - un développement unilatéral valable pour toutes les sociétés -, ne sont pas nécessairement pertinentes ailleurs.

Un petit retour sur l'histoire nous démontre combien les concepts de progrès et de développement se rapportent à des généalogies et visions pleinement occidentales. Une notion comme «tiers-monde» proposée par Alfred Sauvy en 1952, en référence au tiers état de l'ancien régime en France, pour qualifier l'ensemble des pays en « voie de développement » illustre l'ethnocentrisme et l'évolutionnisme implicite de la notion.
Des groupes de recherche divers, comme l'Institut universitaire d'études du développement de Genève par exemple, questionnent depuis des années la notion de développement au sens occidental. En effet, si les processus linguistiques de catégorisation sont passés de la " colonisation » $\mathrm{au}$ " développement » et, plus récemment, à la « mondialisation », les principes sous-jacents de l'humanisme occidental, si bien démontés par Michel Foucault, restent les mêmes. Seules les emphases et justifications changent historiquement : conversions religieuses, transferts de technologies, économie de marché, etc. Récemment, la dimension localisée du "développement » a cependant été soulevée dans plusieurs études qui stigmatisent les propos positivistes qui lui sont attachés (Rist, 1996; Hooper, 2000). Il n'en demeure pas moins que, hors des cercles académiques, le « développement » reste la référence et le « non-développement», ce qu'il faut dépasser. Face à ces réflexes ethnocentriques, la réflexion sur les « freins et atouts du développement » ici ou là se doit de replacer les choses dans leur contexte : de quel développement parle-t-on ? À quel système de valeurs et modèle de société se rapporte-t-il ? Pourquoi et pour qui est-il mis en œuvre?

Si la distanciation avec le concept de développement sous-tend la grande majorité des travaux sociologiques dans ce domaine, il n'en reste pas moins vrai qu'il est difficile de porter sur lui un regard critique en l'évacuant de nos réflexions. Cela est aussi valable pour la notion de "transfert de technologie" qui est l'un des facteurs constitutifs des processus de développement (Geslin, 2004). Ces transferts ont fait l'objet de nombreuses analyses sociologiques et anthropologiques. Celles-ci ont su en révéler les mécanismes et en pointer les échecs, mais elles n'ont toutefois jamais convaincu les instances internationales qui persistent encore aujourd'hui à utiliser l'expression «transferts de technologies » de façon positiviste dans leurs considérations et plans d'actions. Sans nous défaire totalement de la notion de "développement », avec quelques décennies de recul et à la lumière des champs disciplinaires actuels, il nous semble important de susciter une réflexion sur ce qu'elle recoupe aujourd'hui sur le terrain polynésien. Après d'autres (Baré, 1987, 1992), nous allons tenter d'explorer dans cet essai les modalités et les limites du développement entendu au sens occidental en Polynésie (française) et quelques catégories polynésiennes du développement afin de voir en quoi elles peuvent diverger des modèles français. Le regard anthropologique proposé ici "[...] suppose que l'on traite le "développement" 
comme un ensemble de formes socio-historiques et culturelles spécifiques [...] » (Baré, 1987 : 269). Ce petit état des lieux du développement, tel qu'il a été jusqu'à présent opéré en Polynésie, permet par ailleurs de pointer la nécessité d'étudier sérieusement les formes sociales d'appropriation idéelles et matérielles pas défini des processus en jeu. On ne peut nier «l'occidentalisation du monde », mais on ne peut pas nier non plus les variabilités de ce processus (Baré, 1995 : 150). Ces variabilités incitent à considérer la relation qui s'inscrit entre des communautés d'acteurs d'horizons culturels différents et, de fait, à prendre en compte les décalages et parfois les perturbations qu'elles génèrent.

Le progrès technologique, l'industrialisation, l'administration, etc., largement importés en Polynésie française, restent des options ou des modalités occidentales, même si l'histoire contemporaine marquée par la valorisation du pouvoir économique semble leur donner une valeur de modèle universel. Du coup, le cas de la Polynésie française est tout particulièrement intéressant pour appréhender, par le biais du regard anthropologique, les conceptions divergentes du développement. Si la présente analyse stigmatise des malentendus et des " échecs », son objectif ultime est avant tout de pointer la nécessité d'étudier sérieusement les formes sociales d'appropriation idéelles et matérielles des processus en jeu.

\section{Développement en Polynésie française}

Toujours animée par la volonté de « développer » ses anciennes colonies, la France a créé un certain nombre d'institutions du développement en Polynésie depuis le début du XVIII ${ }^{\mathrm{e}}$ siècle. Le développement est d'ailleurs allé de pair avec la colonisation, ainsi justifiée par un discours humaniste. Dans une logique développementaliste, la France a investi dans ce territoire d'Outre-mer (et continue de le faire en situation néo ou post-coloniale, selon la perspective) en matière d'économie, de santé, d'éducation, de consommation, de tourisme, de transport, d'emploi, d'artisanat, etc. Le gouvernement du territoire polynésien s'est lui aussi engagé depuis vingt ans dans une logique du développement. Le projet de loi organique, présenté devant le Sénat le 22 octobre 2003, portant statut d'autonomie de la Polynésie française, affiche claire- ment que le développement constitue un objectif politique en soit :

« La République garantit l'autonomie de la Polynésie française ; elle favorise l'évolution de cette autonomie, de manière à conduire durablement la Polynésie française au développement économique, social et culturel, dans le respect de ses intérêts propres et de l'identité de sa population. » (Sénat ${ }^{1}, 22$ octobre 2003)

Le « pacte de progrès » négocié entre le territoire et l'État depuis la fin des essais nucléaires en 1996 a permis le transfert d'importants fonds financiers. Comme pour les autres collectivités territoriales françaises d'outre-mer, on prévoit que ce transfert sera pérennisé pour une période indéfinie. Dans ce processus de développement initié par l'État puis par le territoire, le destin des cinq archipels de la Polynésie devient irrémédiablement lié aux politiques conçues depuis Tahiti. Le territoire s'est engagé dans la construction d'aéroports, de ports dans les archipels, dans la modernisation de l'île de Raiatea (îles Sous-leVent) pour en faire une « métropole » d'équilibre par rapport à Tahiti. Partout, des réseaux routiers et électriques sont développés. Des « plans stratégiques » sont élaborés pour accroître le tourisme, l'agriculture, la pêche et pour attirer les retraités de l'hexagone français en Polynésie, afin qu'ils réinjectent localement l'argent qu'ils reçoivent du gouvernement français. Selon les projets gouvernementaux en Polynésie, les îles Sous-le-Vent auraient vocation à devenir un pôle d'attraction touristique ayant pour centre l'île de Bora Bora qui accueille déjà la moitié des touristes en Polynésie. L'archipel des Marquises deviendrait une base de pêche ; celui des Australes, le «grenier» de la Polynésie ; les Tuamotu auraient vocation à développer la perliculture et le tourisme, tandis que Tahiti et Moorea demeureraient le centre administratif, logistique et financier de la Polynésie française.

Le cas de Rapa est exemplaire en ce sens qu'il rend compte d'une bonne partie de la situation des îles localisées aux Australes, aux Tuamotu de l'Est et aux Marquises du Sud. Dans cette petite île de $40 \mathrm{~km}^{2}$ de l'archipel des Australes, le développement (qui est aussi la globalisation) est médiatisé par un bateau tous les deux mois environ. Dans ce contexte, tout ce qui arrive est plus ou moins absorbé par les quelques quatre cent cinquante habitants, à leur rythme et selon leurs modes de pensées et d'appréhension des choses. Des aspects évidents du développement au sens classique se sont jusqu'à présent exprimés par la

1. Annexe au procès-verbal de la séance du 22 octobre 2003 du sénat : projet de loi organique portant statut d'autonomie de la Polynésie française, présenté au nom de M. Jean-Pierre Raffarin, Premier ministre, par $\mathbf{M}^{\mathrm{me}}$ Brigitte Girardin, ministre de l'Outre-mer. 
mise en place d'un groupe électrogène qui fonctionne en permanence en alternant sur deux moteurs, un câble de un kilomètre de long qui passe au fond de la baie principale pour relier les deux villages de l'île, l'électricité qui permet l'usage du téléphone et de la télévision, le système d'approvisionnement en eau, la construction d'un petit port pour permettre aux bateaux de décharger des marchandises, d'une route reliant le port aux deux villages, une école, une mairie, une infirmerie, deux temples protestants, des maisons préfabriquées (dites MTR), quelques voitures, des bateaux à moteur, etc. L'idéologie du développement apparaît également dans la plantation, décidée par le ministère de l'Agriculture du territoire il y a près de trente ans, de plusieurs forêts de pins dans les montagnes de l'île afin de les exploiter plus tard mais qui, en absorbant année après année l'eau des sols, ont repoussé les tarodières traditionnelles au fond de la baie principale ou dans des baies assez éloignées des villages, au grand désarroi des anciens. Cette idéologie est enfin en jeu dans le projet de construction d'un aéroport dans l'île par le territoire (projet auquel la majorité de la population s'oppose aujourd'hui car elle y voit un seuil irréversible dans la situation de relative autonomie de l'île).

\section{Développement unilatéral et...}

\section{...mal développement}

Un problème associé au développement importé en Polynésie est celui de la dépendance qu'il engendre, avec la France notamment. Cette dépendance générée par les transferts de fonds a engendré une société de consommation qui semble aujourd'hui servir à Tahiti de "projet de société ». Le gouvernement territorial, qui détermine les grandes orientations et entreprend les actions politiques, s'est résolument engagé dans une politique développementaliste : salaire pour tous et consommation à outrance, bref un projet de société véhiculé par les élites qui ne tient pas compte des réalités locales et des notions endogènes.

Les transferts de fonds destinés au développement des infrastructures et institutions en Polynésie française n'aboutissent d'ailleurs pas toujours dans leur totalité là où ils sont destinés. Des constructions prestigieuses et ostentatoires sont ainsi programmées par des représentants du territoire comme le Palais présidentiel, la mairie de Papeete, de Pirae, sans compter les nombreux bénéfices personnels de personnes faisant usage des institutions qui les emploient sans s'investir dans leur fonction, ou détournant à leur profit le bien public. Ces pratiques ne sont bien sûr pas spécifiques au territoire polynésien mais nous avons ici un héritage du développement programmé dans des contextes culturels ou le sens civique repose sur des modèles divergents. Paradoxalement, l'augmentation des transferts financiers entre la métropole et la Polynésie a favorisé le développement local des inégalités sociales. Certains fonctionnaires territoriaux et d'État voient leur salaire majoré grâce à un cœfficient multiplicateur allant de 1,5 à 2,4 par rapport à leur salaire en métropole, tandis que les salaires les plus bas qui concernent la majorité de la population atteignent tout juste $80 \%$ du salaire minimum métropolitain.

À Tahiti, on entend souvent dire que la population qui profite le plus du développement est celle des demis (àfa), des Chinois (tinito) et des métropolitains (farāni) plutôt que celle dont le mode de vie est plus modeste et rural et dont les souches polynésiennes sont plus évidentes (taata $m a \bar{a} h i)$. Soulignons également le fait que ces manières de catégoriser les composantes de la société polynésienne ont pris le sens qu'on leur prête aujourd'hui essentiellement par rapport à une idéologie du développement. À l'origine, les « demis » (taata àfa) désignaient les descendants des blancs qui s'étaient unis avec des fils ou des filles de chefs : assignation identitaire autant que statutaire. Aujourd'hui, les principaux bénéficiaires et initiateurs du "développement importé » sont précisément ceux-là même qui ont contribué à faire évoluer les caractéristiques attachées à cette dénomination de "demis". Dans la Polynésie d'aujourd'hui, la notion de « demis » fait moins référence à un descendant de chef et d'Européen qu'à un individu métis (quels que soient les sangs d'origines) qui a su bien se placer par rapport à l'administration, au commerce et à la logique de développement. Autrement dit, le développement, par les pratiques socio-économiques qu'il détermine et les acteurs qu'il implique, contribue à faire évoluer des désignations sociales, politiques et ethniques.

Le développement programmé dans les îles de la Polynésie française est par ailleurs différent de celui mené à Tahiti. Très souvent, les habitants et municipalités des îles sont obligées de s'ajuster aux orientations politiques du gouvernement du territoire sous peine de se voir « fermer les robinets » selon l'expression locale.

Un des exemples le plus parlant d'un mal développement est sans doute le cas de l'île de Bora Bora. Médiatiquement et mondialement connu pour avoir abriter une base américaine 
durant la Seconde Guerre mondiale, cette île a connu une croissance extraordinaire de l'industrie touristique de luxe. Désormais, les plages ou les îlots réservés à une clientèle internationale ne sont plus accessibles aux autochtones. Dans la majorité des cas, l'industrie étant aux mains de multinationales, les profits générés ne sont pas réinvestis dans les activités locales, mais repartent vers d'autres destinations. Pour permettre le développement de cette filière, il a fallu l'importation massive d'une main-d'œuvre salariée de Tahiti ou des autres îles de l'archipel. Cet afflux de population a engendré des conflits désormais réguliers entre population locale et « importée ». Conscientes des déséquilibres générés par le développement, les autorités locales ont décidé de limiter les installations d'hôtels, sans pour autant remettre en cause la logique sous-jacente au «mal-développement». C'est en effet officiellement le «tourisme riche » qui est encouragé par les autorités politiques locales à Tahiti, au détriment d'un tourisme rural, plus modeste et à moins grande échelle, mais impliquant plus la population polynésienne dans son ensemble.

Dès lors que nous passons d'une échelle archipélagique à une échelle locale et quotidienne, les moteurs, l'essence, les lignes en plastique, les hameçons en métal, les fusils sous-marins, etc., pour la pêche par exemple, fournissent des moyens d'approvisionnement plus faciles que les moyens traditionnels mais aussi plus coûteux. Les $4 \times 4$ permettent aussi des déplacements routiers plus aisés pour la minorité qui peut se les offrir. Ces nouveaux éléments du quotidien facilitent indéniablement la vie de tous les jours (ce qui explique leur succès), mais ils s'inscrivent dans des modèles de consommation euroaméricains quelque peu décalés en Polynésie au regard des conditions climatiques pour lesquelles ils n'ont pas toujours été conçus et des capacités de maintenance localisée de ces engins. D'une part, la nature ne permet pas véritablement leur conservation (moteurs qui rouillent, artefacts de toute sorte qui pourrissent, etc.), d'autre part, leur importation comme allant de soi ne correspond pas véritablement aux besoins quotidiens des populations, notamment dans les îles. À Tahiti, l'extension du parc routier pose par ailleurs déjà depuis plusieurs années de gros problèmes de congestion du trafic. Il est aussi intéressant de relever la présence de la télévision câblée. Il ne s'agit pas ici de remettre en cause son usage dans les îles polynésiennes, mais de rappeler qu'elle apporte des informations et des divertissements qui ont leur origine et leur pertinence culturelle ailleurs. Bien entendu, les populations sujettes aux transferts de technologies ne reçoi- vent jamais ces transferts de façon passive. Elles opèrent des ajustements et des reformulations dans leurs gestions de ceux-ci. Ces formes sociales d'appropriation, qui font parfois l'objet d'études passionnantes, ont aussi lieu en Polynésie française. Elles mériteraient des études spécifiques que nous ne pouvons entreprendre dans le cadre de cet article.

L'ethnocentrisme est toujours sous-jacent au développement version unilatérale. Voici un exemple frappant de décalage dans la définition des besoins. Nous avons assisté à la première liaison par hélicoptère Tahiti-Rapa via Raivavae (aux Australes) pour ouvrir la ligne et permettre la possibilité d'évacuations sanitaires urgentes vers Papeete. Un Super Puma s'est ainsi posé un après-midi de septembre 2001 sur le terrain de football du village de Haurei. Neuf militaires français gradés en sortirent et furent accueillis par une partie de la population. À peine posé le pied sur le sol, l'un d'entre eux nous confia en aparté sa grande impression face à la majesté de l'île, mais ajouta aussitôt que celle-ci « ne pourrait pas s'en sortir sans l'internet »... Cet épisode quelque peu caricatural n'en révèle pas moins des représentations normatives extérieures sur ce qui est bon pour les îles sans prendre en compte leurs spécificités et leurs réels besoins internes.

\section{...inadéquations culturelles}

L'importation de modèles extra polynésiens, sans véritablement envisager leurs adéquations avec les systèmes de représentations et les pratiques polynésiennes, engendre des problèmes nouveaux en Polynésie française. L'usage relativement récent dans les îles du moteur pour aller à la pêche a ainsi changé beaucoup d'habitudes et recomposé des pans très spécifiques de la société. Outre le coût financier plus important pour s'approvisionner avec l'essence, la dimension communautaire de l'activité a largement disparu. À Rapa par exemple, on se souvient des pêches collectives, à six hommes sur une baleinière, à la fin desquelles le poisson était distribué entre tous les pêcheurs. Ce partage collectif permettait à chacun d'avoir du poisson. Aujourd'hui, le moteur facilite les sorties individuelles en mer et les plus âgés, qui ne sont plus physiquement en mesure d'aller seuls à la pêche, doivent désormais compter sur la générosité des plus jeunes pour avoir leur part de poisson. Fort heureusement dans cette île, les habitudes communautaires n'ont pas disparu et les poissons sont encore largement distribués autour de soi au retour de la pêche (la notion màòhi d'ailleurs constamment évoquée à Rapa pour valoriser 
l'entraide et le partage communautaire est opere). Il n'en reste pas moins que l'apparition du congélateur permet aux pêcheurs de stockeret donc de conserver pour leur usage propre - le fruit de leurs pêches.

Le développement du système éducatif en Polynésie française selon les modèles français a aussi considérablement transformé les pratiques et les connaissances. Si apprendre l'histoire de France et de l'Europe, à l'école primaire comme à l'université, apparaît comme un facteur d'ouverture, ni le modèle d'une éducation laïque à la française, ni celui d'une école privée et religieuse (principalement catholique et protestante) ne permettent une réappropriation de l'histoire polynésienne souvent méconnue de la population scolaire. Il est indéniable que depuis quelques dizaines d'années les établissements d'enseignements privés ont fait des efforts considérables dans l'apprentissage de la langue tahitienne ou dans l'apprentissage de l'histoire locale. Mais ce qui reste en cause est bien le modèle d'enseignement patriarcal et occidental dont les contenus ne sont jamais remis en cause ou ne font pas l'objet d'un examen critique. Le non-enseignement (qui traduit une ignorance volontaire) des pratiques et des connaissances culturelles et historiques océaniennes est source de malaise identitaire et culturel. Longtemps considérées comme des obstacles à l'idéologie du développement, ces connaissances sont tout juste évoquées dans le cadre d'un cycle professionnel (Centre des métiers et de l'art) ou plus récemment à l'université de la Polynésie française (cours en civilisation polynésienne), mais restent dévalorisées par rapport au parcours considéré comme classique.

C'est d'ailleurs ce « parcours classique » valorisé à Tahiti qui va, dès onze ans, extraire les enfants de leur famille et de leur île pour aller en internat dans le collège de leur île chef lieux. Les élèves de l'archipel des Australes doivent ainsi entrer au collège à Rurutu ou Tupuai après l'école primaire ; ceux des îles Sous-le-Vent vont à Raiatea tandis que les enfants des Tuamotu et des Marquises doivent souvent se rendre à Tahiti. Cette situation de rupture (sauf lorsque l'un des parents quitte temporairement le foyer conjugal et accompagne l'enfant dans son nouveau lieu de scolarité) n'est pas sans causer des perturbations émotionnelles et psychologiques car les enfants et adolescents ne reviennent chez eux que deux fois dans l'année pour les fêtes. S'ils poursuivent leur scolarité, ils vont ensuite à Tahiti. Autrement, ils reviennent dans leur île d'origine, bien souvent en situation d'« échec scolaire » selon les critères imposés. Les spécia- listes invoquent quelques raisons " objectives" pour expliquer cet échec scolaire : un milieu familial qui ne prépare ni n'encourage l'enfant à être performant à l'école ; une mauvaise maîtrise de la langue française due au milieu de vie; des redoublements successifs inefficaces qui découragent l'enfant, etc. Chacune de ces raisons repose sur un modèle d'éducation importé. Or, la langue māòhi possède deux concepts pour désigner les stades d'immaturité et de maturité au sens polynésien : le taureàreà (l'adolescent en âge ou en esprit) qui commet des erreurs de jeunesse et le taata paari (la personne sage) qui assume ses responsabilités. Ces représentations ne s'accordent pas avec celles occidentales de l'échec scolaire car un taata paari peut avoir une mauvaise scolarité. De même, certaines personnes demeurent taureàreà au delà de vingt-cinq ans et, sur la base de cette catégorie, il leur est accordé une place dans leur environnement culturel.

Le développement importé en Polynésie française a également des conséquences importantes et préoccupantes en matière d'alimentation. Les pratiques alimentaires des populations locales ont en effet beaucoup changé avec des consommations de nouvelles nourritures européennes (souvent très sucrées), pas véritablement appropriées au climat tropical et qui, lorsqu'elles sont associées aux aliments plus traditionnels (fruit de l'arbre à pain, taro, etc.) engendrent de très nombreux cas d'obésité.

L'idéologie du développement et du progrès médical a paradoxalement créé de nouveaux problèmes de dépendance des populations vis-àvis de Tahiti. Le développement des soins médicaux modernes pour tous a considérablement amélioré la santé des Polynésiens, et ceci à grands frais, notamment lorsque des évacuations sanitaires vers Tahiti sont en jeu. La logique de la sécurité en matière de santé a toutefois engendré depuis quelques années des situations très complexes et pour certains très pénibles. L'obligation juridique d'aller accoucher dans les hôpitaux de Tahiti ou de Raiatea, ces deux îles ayant les seuls hôpitaux habilités à procéder à des accouchements en Polynésie française, conduit les futures mères à se rendre dans ces îles (principalement Tahiti) avant qu'elles atteignent le septième mois de leur grossesse afin de prévenir tout risque lors de l'accouchement. Ce système sécuritaire a pour conséquence pratique que plus aucun enfant ne naît (ou est censé nâ̂tre) dans les îles et que tout le monde naît donc désormais officiellement « tahitien », ce qui n'est pas sans causer des perturbations dans une culture où l'attachement symbolique et matériel à la 
terre ancestrale est profond. Cette situation engendre des difficultés financières pour demeurer quelques mois à Tahiti, en faisant notamment jouer son réseau de fetii (apparentés). Dans bien des cas, les aides octroyées par la Caisse de sécurité sociale à Tahiti demeurent la seule ressource financière pour faire face aux dépenses liées à ces déplacements. Dans ce nouveau contexte, des pratiques culturelles anciennes perdurent toutefois à travers des ajustements (comme cela est souvent le cas) lorsque le cordon ombilical (le pito) est ramené par la mère dans un bocal pour l'enterrer dans l'île d'origine.

\section{Attitudes culturelles différentes}

Mis à l'épreuve de la Polynésie, le concept de développement ne coïncide pas avec bon nombre de discours, de catégories et de pratiques locales. Il convient de partir d'une évidence : hors du registre politique ou administratif, le terme « développement »n'est jamais utilisé par les populations. Si on pousse nos interlocuteurs à expliciter leur pensée, ils disent qu'il s'agit là de préoccupations popaa (étrangères) qui ne les concernent pas.

Un malentendu évident, entre le développement au sens occidental et le développement selon une approche polynésienne, réside dans la traduction même du terme. Faahotu (développer), faahoturaa (développement) renvoient d'abord à l'image d'un fruit que l'on fait croître, d'un arbre ou d'une plante qui produit des fruits. En tant que substantif, ce terme n'est jamais employé seul comme en français. Par exemple, si quelqu'un dit te faahoturaa (le développement), ses interlocuteurs se demandent aussitôt de quoi ? Le développement n'a donc aucun sens tant qu'il n'est pas relié à une pratique précise ou à une image évocatrice. $\mathrm{Si}$ on insiste tout de même en voulant signifier en tahitien le " développement $\gg$ au sens occidental, on dira alors (une variante possible) : te faahoturaa i te maitai i nia $i$ te fenua (le développement du bien sur la terre / dans le pays). Ainsi, le développement n'est jamais entendu en dehors d'une morale politique, religieuse ou économique. C'est précisément cette morale sous-jacente, cette communauté d'idées, de valeurs et d'idéologies que nous voulons pointer ici.

Un autre mot proche de celui de faahoturaa, dont le sens est intéressant à souligner, est le mot tupuraa (croissance). Au sens polynésien, la croissance (tupuraa) traduit notamment l'extension d'un réseau généalogique et la circulation du mana (pouvoir). L'augmentation du réseau généalogique, sa profondeur et la circulation de la richesse dans le réseau colorent l'idée de croissance et de développement. Les cosmogonies polynésiennes et les références régulières aux généalogies montrent en effet que le monde est envisagé comme un vaste réseau généalogique perpétuellement en extension et en croissance $\left(\right.$ tupuraa $\left.^{2}\right)$. Aussi, du point de vue polynésien, le monde est perpétuellement «en développement $\gg$ et cela ne dépend pas des niveaux affichés de PNB, de longévité de la population ou de taux de fécondité. De manière corollaire, hors du réseau généalogique, point de développement. Dans cette perspective, ce qui fait la force d'un réseau, c'est précisément son développement continu. Le mana et la richesse qui y est associée doivent circuler car ni les dieux ni les chefs n'en ont le monopole. La redistribution immédiate de la richesse passe par une circulation perpétuelle de celle-ci.

Comme dans d'autres situations en Océanie, ces quelques idées soulignent une dimension importante du développement au sens polynésien : son caractère collectif et communautaire, sa démarcation avec un développement créateur d'inégalités sociales et culturelles. Prenons quelques exemples.

Le rapport au profit s'opère dans une logique toute particulière en Polynésie, logique qui laisse perplexe beaucoup de non-Polynésiens qui y sont confrontés. Une situation tout à fait caractéristique de conceptions divergentes du travail et du profit est le cas de la pêche. Les Polynésiens ont pour habitude de pêcher ce dont ils ont besoin et ce qu'ils veulent vendre. Si un acheteur non polynésien veut passer une commande de poisson plus grande que ce que le pêcheur à l'habitude de pêcher, il engendrera souvent une réaction qui n'entre pas dans sa logique : plus il achètera de poisson, plus il paiera cher chaque poisson. Pourquoi ? Tout simplement parce que cela demande plus de travail au pêcheur qui ne l'envisage pas comme un gain général pour lui. On observe le même phénomène dans le cas de l'artisanat où les productions sont ajustées avant tout par rapport à une demande familiale et villageoise plutôt que commerciale. Les grandes fêtes religieuses seront l'occasion pour les femmes de former des groupes de travail (pupu òhipa) afin de préparer des colliers de coquillages, de coudre des couvertures arborées de motifs tradi-

2. Sur l'analyse des cosmogonies hawaiienne, māòhi et tahitienne fondatrices, voir la thèse de Bernard Rigo (2002). Les entités humaines et non humaines, les humains, les dieux, les pierres s'engendrent les uns les autres dans une copulation cosmique. Les dieux ne sont même pas à l'origine du monde qu'ils n'ont pas créé. 
tionnels (tifaifai) en vue de la distribution de ces présents aux dignitaires que l'on veut honorer. Mais toute surcharge de travail régulière dans la perspective d'une commercialisation se traduit par la fixation d'un prix quasi exorbitant et rédhibitoire. En Polynésie comme dans d'autres cultures, le profit est plus un rapport, une relation qu'une quête individuelle. Il revêt un caractère collectif et ne peut être désolidarisé des relations proches (bénéficiant d'abord à la famille, à la communauté et au voisinage).

Dans bien des discussions individuelles, le rapport social au profit est envisagé comme un jeu qu'on essaye de gagner et dont la fin est prévue d'avance. L'expression tāmata noa (essayer seulement, tenter sa chance) lorsqu'un Tahitien parle d'une activité qu'il souhaiterait démarrer, exprime bien cette idée de jeu. Il s'agit de « s'amuser » (ârearea) pendant un temps donné, de «faire un coup » et d'en profiter sans vraiment penser à différer la redistribution des fruits (au sens polynésien) ou à investir dans une optique capitaliste. Paradoxalement, même l'agriculture commerciale de pommes de terre et de carottes à Tupuai est envisagée comme un jeu qu'on essaye de jouer pendant une ou deux saisons ou tant qu'on gagne. Ce type d'agriculture est d'autant plus prisé que les périodes de production sont courtes (trois mois). Cette agriculture commerciale participe en réalité d'une polyactivité où l'espace-temps de la famille élargie $(\hat{o} p \bar{a})$ se partage entre la pêche, la tarodière, les emplois administratifs temporaires et un peu d'agriculture.

La limitation des besoins et la focalisation sur l'immédiatement utile ou pertinent dans son «monde de vie » semble être un aspect important des cultures polynésiennes. D'autant que, comme nous l'avons vu, le profit personnel est d'abord envisagé dans la relation qui lie l'individu au groupe. On retrouve cette attitude dans l'agriculture qui est avant tout de subsistance. On entend ainsi souvent l'expression suivante à Rapa : aita moni to ù, e tamaa noa (littéralement : Je n'ai pas d'argent mais on peut toujours manger). Cette expression dénote également le fait que l'agriculture de subsistance, comme dans tout le Pacifique, a une valeur qui va bien au-delà de la simple évocation de "subsistance». Comme le signale Anthony Hooper (2000),
« l'agriculture de subsistance » traduit assez mal l'idée que les sociétés océaniennes associent à cette partie de l'activité sociale. Ce n'est pas seulement la « subsistance » qui est en jeu, mais une grande variété d'échanges et de redistributions réciproques, de valeurs affichées (modestie, altruisme), de moralités et d'idéologies constamment réactualisées dans les rapports sociaux ${ }^{3}$. Dans beaucoup d'archipels de la Polynésie comme ailleurs en Océanie, l'« agriculture de subsistance » est une notion souvent confondue avec la culture ou la coutume. Ainsi, en Polynésie comme dans d'autres cultures non occidentales, les rationalités économiques ne sont pas nécessairement celles qu'on veut y importer. L'économie informelle, fondée sur l'échange de service, reste encore profondément présente dans les relations sociales. Ces attitudes révèlent des relations au travail et au temps particulières et une forme de sécurité relationnelle et juridique assurée par l'institution des familles élargies.

Il est parfois utile de rappeler des évidences : les rapports au temps, à autrui et aux choses reposent également sur des modèles culturels spécifiques. La langue māòhi réunit dans un même mot, ite, les notions de "voir» et de " savoir », conjuguées dans une seule et même conception de la connaissance. Le présent et l'action dans un plein présent dont on fait l'expérience restent des valeurs fondamentales. Tout se passe comme si le savoir au sens polynésien était d'abord perceptif et sensitif, fondé sur l'expérience physique (ou l'expérience proche, la co-présence importe plus que ce qui est loin), ce que l'on peut appeler un sensitive set of knowledge ${ }^{4}$. C'est dans cette logique que doit aussi être comprise la notion de fiu: on décroche physiquement et moralement d'une situation particulière pour se retrancher en soi. Le sensitive set of knowledge ne signifie pas que les Polynésiens n'aient pas de conception spécifique du découpage du temps passé/présent/futur et qu'ils vivent perpétuellement dans le présent ; cela ne signifie pas non plus, comme l'a dit Marcel Mauss, « que les Maoris vivent perpétuellement dans l'univers de la magie » (Mauss, 1968), ni que «le monde maori se développe comme un éternel retour» (Eliade, 1989 : 274). L'analyse linguistique des langues polynésiennes montre qu'aucune distinction a priori n'est faite entre un

3. À cet égard, Anthony Hooper (2000), tenant compte des situations de développement spécifiquement océaniennes, indiquait que le découpage occidental traditionnel entre secteur primaire, secondaire et tertiaire paraissait culturellement moins pertinent qu'un découpage entre « subsistance », public, privé.

4. Nous devons cette idée à Paul Ottino (communication personnelle) qui considérait que, pour comprendre beaucoup d'attitudes polynésiennes, on pouvait prendre la notion phénoménologique de cognitive set of knowledge, qui est parfaitement explicative dans les sociétés occidentales, et remplacer cognitive par sensitive. Le sensitive set of knowledge était pour lui une modalité d'être polynésien. Nous adhérons totalement à cette perspective. 
axe temporel et un axe spatial. Les Tahitiens peuvent dire deux choses différentes d'une même manière, privilégiant tantôt l'aspect temporel, tantôt l'aspect spatial. Si les grammaires occidentales ont choisi de décrire les temps verbaux, la grammaire tahitienne a privilégié les « espaces verbaux ». Te ite nei/nalra n'expriment pas uniquement un temps présent/passé, mais une localisation de l'action dans l'espace. Ce qui est loin pouvant être passé ou futur tandis que ce qui est proche est toujours plus important (voir Saura, 1996 et Tcherkézoff, 1998). Cette conception phénoménologique de la réalité implique des attitudes différentes dans le rapport au temps et à l'espace, attitudes souvent incomprises par une idéologie du développement occidental. Ainsi, les promoteurs du développement ne saisissent pas pourquoi, le futur et la "perspective d'une vie meilleure ", telle qu'ils l'envisagent et avec tous les avantages qu'ils y voient, restent insuffisamment valorisée en Polynésie. De même, ils sont souvent surpris par le fait que les gens prennent leur temps pour parler comme si le présent était infini. On a bien ici affaire à un espace-temps spécifique qui privilégie la co-présence.

Les conceptions polynésiennes de l'espace et de la nature, et de la relation que l'on entretient avec elle, sont aussi différentes de celles qui marquent l'idéologie du développement. Rappelons que l'idéologie occidentale du développement repose sur l'idée que l'homme doit/peut maîtriser la nature pour améliorer ses conditions de vie. Le problème est que l'idée même de contrôle de la nature est étrangère aux représentations locales. L'inscription des relations société/nature dans un continuum a donné lieu à de nombreux travaux anthropologiques, principalement dans les sociétés amazoniennes (voir par exemple Descola, 1996; Guille-Escuret, 1989) et à une moindre échelle dans certains pays d'Afrique (comme Leach et Fairhead, 1994). Dans un ouvrage récent, Bruno Saura (2004) nous offre une analyse de ce lien en Polynésie française. Contrairement à la vision occidentale qui fait de la nature un domaine indépendant qui se définit par son altérité par apport à la culture (Descola, 1996 : 88), la pensée polynésienne envisage la nature et la culture selon un principe de continuité. Cela aboutit à un paradoxe : d'une part, la nature n'est pas pensée en tant que catégorie ontologique séparée de la culture comme en Occident; d'autre part, dans le même temps qu'elle est un « impensée », la nature est au cœur des revendications identitaires et politiques, des discours des intellectuels océaniens dans tout le Pacifique. Dans leur logique, respecter la nature, c'est respecter la terre qui relie aux ancêtres (tupuna) et au monde des dieux. C'est aussi les honorer et reconnaître la place qui est la leur dans le monde d'aujourd'hui.

Par rapport à une problématique de développement (que nous avons dernièrement observé à Tupuai), cela signifie que s'approprier les terres pour l'économie, oblige à une redistribution (formelle et informelle) des fruits du travail pour tous les descendants qui se reconnaissent comme descendant d'un tupuna mau (vrai ancêtre) depuis le tupuna à l'origine de la revendication foncière au $\mathrm{XIX}^{\mathrm{e}}$ siècle. Dans le même sens, l'appropriation individuelle (en fait, au bénéfice d'une famille nucléaire) de l'usage de la terre n'est culturellement tolérée à Tupuai que tant que l'enrichissement qui en découle ne dépasse pas les besoins évidents des bénéficiaires (construire sa maison, acquérir une Vespa ou une bicyclette, acheter une machine-à-laver à une tante, participer aux frais collectifs de mariage et des fêtes religieuses, etc.). Dans le cas contraire, la "mise en valeur des terres» provoque des tensions, des conflits et des jalousies souvent mal compris par les observateurs extérieurs ${ }^{5}$. Ainsi, le malentendu réside dans le fait qu'il n'y a pas à Tupuai de refus obstiné d'une mise en valeur des terres, mais le développement s'exprime localement selon des modalités variées (en fonction des groupes de parenté ou $\hat{o} p \bar{a}$ ) et spécifiques d'accès aux ressources et de redistribution de ces mêmes ressources. Ces modalités, impensées dans une théorie économique de la rationalité individuelle pure, mériteraient plus d'attention. La terre ( fепиа) n'est ainsi traditionnellement pas envisagée comme un bien matériel ou économique pouvant faire l'objet d'une appropriation individuelle au sens du code civil puisqu'une approche patrimoniale semble l'emporter. Elle a été indivise jusqu'aux revendications foncières héritées de l'imposition du code civil français en Polynésie et continue de le demeurer dans la majorité des cas. Outre les enjeux économiques, le rapport

5. Cela est aussi vrai dans les cas de développement autochtone dits « réussis » comme les filières perlières aux Tuamotu, la récolte du coprah aux Marquises ou les filières agricoles aux Australes. Dans ces derniers cas, le développement que l'on observe depuis vingt ans n'a pas aboutit à une individualisation des droits de propriété privée et repose en grande partie sur des réseaux de solidarité communautaires et familiales. Le statut de la «terre indivise », pourtant identifiée comme un « frein au développement » par les élites polynésiennes, a au contraire permis aux familles māòhi les plus modestes d'accéder à des ressources familiales (partie lagonaire attachée à la terre ; terres de subsistance ou affectées à l'agriculture), sans pour autant déposséder les groupes familiaux de la propriété de ce patrimoine. 
avec la terre est notamment un rapport émotionnel et familial dans la mesure où elle met en jeu de l'ancestralité et des rattachements physiques (le cordon ombilical, le pito, de chacun étant enterré dans les terres ancestrales).

La culture polynésienne n'a que très peu mis l'emphase sur le développement de la dimension matérielle des choses. Cela est évident avec les marae, caractérisés par la simplicité de leur structure. De même, on peut constater une relative indifférence des Polynésiens des îles envers ce que les archéologues veulent leur présenter comme leur " patrimoine ». Les forts de Rapa, uniques en Polynésie française, périclitent ainsi jour après jour par manque d'entretien et de protection. Si les discours sur le patrimoine polynésien, pour des enjeux culturels et touristiques, trouvent un certain écho à Tahiti (notamment en milieu urbain), il en va tout autrement dans des lieux où la polynésianité se vit dans un plein présent et n'éprouve pas le besoin de se mettre en scène pour croire qu'elle existe. Il en est de même des revendications identitaires et des discours développementalistes qui, dans l'ensemble, ne sont pas substantialisées comme à Tahiti.

La notion d'efficacité de l'action est elle aussi déterminée par une logique propre. Il est frappant de voir les non-Polynésiens dans les îles critiquer le manque de soin apporté par les Polynésiens aux infrastructures et matériels (véhicules, moteurs, etc.) qui leurs sont fournies dans le cadre de leur " développement ». Dans les îles de la Polynésie française, les travaux publics y sont très lents et plus ou moins « efficaces 》 selon les critères occidentaux. Les complaintes des fonctionnaires représentant le territoire ou de l'État sur le matériel non entretenu, les programmes mal ou pas suivis, etc., sont nombreuses. Il en va ainsi des réseaux hydrologiques, de l'entretien des stations agricoles destinées à la recherche agronomique, etc. Ces attitudes critiques, fondées sur des jugements typifiés et une totale méconnaissance de la culture des personnes incriminées, ne changent pas les pratiques quotidiennes des Polynésiens, si ce n'est le fait de devoir intérioriser la critique publique. À cet effet, il faut souligner le poids de la culture de la culpabilité/culture de la honte (haamā) nouvellement exercé par l'idéologie du développement. L'éducation occidentale et la culture du développement ont en effet souvent mené à une véritable culture de la honte par rapport à des pratiques et des discours considérés comme plus traditionnels ${ }^{6}$.

L'inadéquation des conceptions du temps, de l'espace, des ressources et du profit, engendre parfois des situations insolites en Polynésie française. Prenons un dernier exemple. Dans une logique de protection de l'environnement naturel pour ne pas épuiser leurs ressources, les habitants de Rapa ont instauré un interdit sur la pêche aux filets tout autour de l'île et la pêche au fusil sous-marin dans la baie principale et les deux baies voisines, toute l'année. Cet interdit ou rāhui est marqué par une forme de tapu, posé par les anciens et entériné par le pasteur lors de prières publiques, qui conduit les insulaires à ne pas enfreindre l'interdit sous peine de dangers et/ou de malheurs pour eux et leurs proches. Il n'est temporairement levé, par le biais d'autres prières, effectuées en général par le pasteur, qu'un seul jour dans l'année au moment des fêtes de Noël. Pour quelques heures, les hommes vont alors pêcher facilement une grande quantité de poissons qui sera ensuite distribuée en parts équitables (selon la grandeur des familles) à toute la population de l'île. On peut comprendre le choc des habitants lorsqu'ils s'aperçurent, il y a quelques années, qu'un chalutier tahitien avait discrètement déposé des cages pour piéger les poissons dans une baie de l'île. Leur réaction fut radicale : des plongeurs de Rapa allèrent récupérer ces cages et les confisquèrent. Souvenir d'une réaction commune et solidaire face à la tentative de prélever sournoisement leurs ressources sans leur autorisation pour une logique de marché qui n'est pas la leur, les habitants font aujourd'hui allusion à ces cages comme un «trésor de guerre ». Le problème juridique auquel ils peuvent cependant faire face est que la pêche, même sous une forme à laquelle ils ont eux-mêmes renoncé pour préserver leur environnement, est autorisée pour les bateaux du territoire polynésien autour de leur île qui se trouve dans les « eaux polynésiennes ».

6. Les analyses de Tosivo Nakayama, de Chuuk (en Micronésie), posent bien ce problème que l'on retrouve en Polynésie française. Il dénonce ce qu'il appelle « l'intimidation culturelle » dont le but est d'amener les Micronésiens à avoir honte de leur façon de vivre, de leurs coutumes et de leurs traditions. À cet égard, la religion du développement que l'Occident a propagé au $\mathrm{XX}^{\mathrm{e}}$ siècle est comparable au christianisme répandu au XIX ${ }^{\mathrm{e}}$ siècle par les missionnaires. Dans les deux cas, on prétendait racheter les peuples insulaires et les délivrer d'un mal intrinsèque après avoir suscité en eux un profond sentiment de mépris de soi. Aux yeux du christianisme, les cultures traditionnelles étaient spirituellement marquées par le péché. De même dans l'économie du développement, ces cultures, telle une épine dans le pied, constituent une entrave au progrès des sociétés peu évoluées, établies dans des îles « lointaines » et insignifiantes, dépourvues des ressources et des compétences du « développement », dans Marshal Sahlins (2000: 22). 


\section{Conclusions}

$\mathrm{Au}$ terme de cet essai, nous espérons avoir pu attirer l'attention, non pas sur une opposition stérile développement occidental (matériel, économique, etc.) / non-développement polynésien, mais sur l'aspect non unilatéral du développement en Polynésie française et sur les formes qu'il revêt dans cette partie du monde. Un dilemme souvent rencontré par les responsables locaux, notamment en milieu rural, est de concilier le développement, au sens occidental, avec la logique collective (très marquée en Polynésie), plutôt que pour des finalités individuelles et privées. Au même titre dans d'autres lieux, depuis plus de cinquante ans, le bilan réalisé par les sciences sociales, et principalement par la sociologie et l'anthropologie, insiste sur le caractère incontournable de la prise en compte des dimensions contextuelles et des spécificités locales. Un grand nombre de méthodologies ont vu le jour dans ce domaine, redonnant sur le papier la parole aux populations, revisitant leurs besoins, prenant en compte les dimensions culturelles et sociales $^{7}$. Leur impact est pourtant resté très faible dans un monde où l'action est souvent privilégiée au détriment d'approches de recherche plus fondamentale, toujours plus coûteuses en temps. Notre intention n'est pas ici de discréditer les transferts de connaissances qui ont de tout temps enrichi les sociétés, mais de se demander quelles sont les meilleures conditions de ceux-ci. Lorsque les particularités locales ne sont pas prises en compte, il est légitime de se demander quels sont les enjeux du développement et à qui il profite vraiment.

Tout se passe comme si on opérait en Polynésie française un «bricolage développemental» en recollant les morceaux importés avec du sparadrap (également importé). Un développement véritable peut-il faire l'économie de penser les concepts utilisés? La Polynésie française possède des avantages certains dans la mesure où elle n'est pas directement soumise aux règles du Fonds monétaire international et de la Banque mondiale et a, en principe, une marge d'autonomie sur la définition de ses choix. Il est profondément regrettable que cette marge n'ait, jusqu'à présent, pas été politiquement utilisée en prenant en compte les logiques polynésiennes du développement.

Faut-il et peut-on revoir la logique du développement en Polynésie selon les catégories polynésiennes? Nous n'avons proposé ici que quelques pistes de réflexion sur les politiques culturelles de développement en Polynésie. Celles-ci doivent à notre sens être poursuivies bien au-delà d'un article car le sujet dépasse largement la question des finalités économiques, et ceci en Polynésie comme ailleurs.

\section{BIBLIOGRAPHIE}

ACADÉMIE TAHITIENNE, 1999. Dictionnaire TahitienFrançais. Fa'atoro parau tahiti-farāni, Papeete, Fare Vāna'a.

Balandier Georges, 1957. Afrique ambiguë, Paris, Plon, $291 \mathrm{p}$.

BAMBRIDGE Tamatoa, (à paraître). Les revendications foncières dans l'archipel des îles Australes, 455 p.

BAmbridge Tamatoa, Jean Pierre Doumenge, Bruno Ollivier, Jacky Simonin et Dominique Wolton (éds), 2002. La France et les Outre-Mers. L'enjeu multiculturel, Hermès 32-33, $634 \mathrm{p}$.

BAMBridge Tamatoa et Christian GHASARIAN, 2002. Juridiction française et droit coutumier à Rapa. Les enjeux d'une traduction, Droit \& cultures 44, pp. 153-181.

BARÉ Jean-François, 1987. Pour une anthropologie du développement économique, Études rurales 105 106, pp. 267-298.

_, 1992. L'économie décrite en tahitien. Quelques remarques, L'Homme 121, XXXII, 1, pp. 143-164.

-, 1995. Les applications de l'anthropologie, Paris, Karthala.

Brugener Pierre, Jacques Grinevald, Fabrizio Sabelli, Eduardo Rallo et Gérald Berthoud, 1975. La pluralité des mondes : théories et pratiques $d u$ développement, Paris-Genève, Presses universitaires de France-IUED.

COMEliau Christian, 2000. Les impasses de la modernité : critique de la marchandisation du monde, Paris, Seuil.

Cowen M.P. et R.W. Shenton, 1996. Doctrines of development, London and New York, Routledge.

ÉCologiste (L'), 2001, Défaire le développement, refaire le monde, L'Écologiste 2, 4, $\mathrm{n}^{\circ}$ spécial 6.

Descola Philippe, 1996. Constructing Nature : Symbolic Ecology and Social Practice, Nature and society. Anthropological perspectives, Ph. Descola and G. Palsson (eds.), London and New York, Routledge, p. 88.

ÉLIADE Mircea, 1989. Mythes, rêves et mystères, Paris, Gallimard, Folio-Essais.

GESLIN Philippe, 1999. L'apprentissage des mondes. Une anthropologie appliquée aux transferts de tech-

7. Le lecteur trouvera une bonne synthèse de ces travaux dans Jean-François Baré (1995), Norman Long (1989) et Jean-Pierre Olivier de Sardan (1995). 
nologies, Paris, Maison des sciences de l'homme, $254 \mathrm{p}$.

_ , 2004 (sous presse). La mise à l'épreuve. Connaissances médiatrices et objets intermédiaire dans les relations sociétés-nature. Un point de vue anthropologique, Le transfert des connaissances en questions. Débat scientifique, débat démocratique, $\mathrm{Ph}$. Geslin et al., CNRs-Éditions.

Guille-Escuret Georges, 1989. Les sociétés et leurs natures, Paris, Armand Colin.

Hobart M. (ed.), 1993. An anthropological Critique of development, London and New York, Routledge.

Hoben Allan, 1982. Anthropologists and Development, Annual Review of Anthropology 11, pp. 349375.

Hooper Antony, 2000. Culture and Sustainable Development in the Pacific, lieu?, Asia-Pacific Press, $227 \mathrm{p}$.

Hyden Goeran, 1980. Beyond Ujamaa in Tanzania: underdevelopment and an uncaptured peasantry, London, Ibadan, Heinemann, 270 p.

JACOB Jean-Pierre, Fabrizio SABELli et al., 1984. Crise et chuchotement : interrogations sur la pertinence d'un concept dominant, Paris-Genève, Presses universitaires de France-IUED.

LeACH M. et J. FAIRHEAD, 1994. Natural resource management : the repoduction and use of environmental misinformation in Guinea's forest-savanna transition zone, IDS Bulletin 25, 2, pp. 81-87.

LockWOOD Victoria, 1993. Welfare state colonialism in rural French Polynesia, Contemporary Pacific Societies: Studies in Development and Change, V. Lockwood, T. Harding, and B. Wallace (eds.), Englewood Cliffs, Prentice-Hall, pp. 81-97.

_, 1993. Tahitian transformation: gender and capitalist development in a rural society, Boulder, Lynne Rienner publishers.

LoNG Norman, 1989. Encounters at the interface. A perspective on social discontinuities in rural development, Wageningen Studies en Sociology, 276 p.
Mauss Marcel, 1968. Sociologie et anthropologie, Paris, Presses universitaire de France.

NDIONE Emmanuel, 1992. Le don et le recours : ressorts de l'économie urbaine, Dakar, Enda, 210 p.

Nyerges A. E., 1996. Ethnography in the reconstruction of African land use histories: Sierra Leone Example, Africa 66, 1, pp. 123-144.

Olivier de Sardan Jean-Pierre, 1995. Anthropologie et développement, Paris, Karthala.

Rigo Bernard, 2002. Altérité polynésienne et conscience occidentale, thèse d'anthropologie culturelle, Nouméa, université de la Nouvelle-Calédonie.

Rist Gilbert (éd.), 1994. La culture otage du développement ?, Paris, L'Harmattan/EADI, L'homme et la société.

Rist Gilbert, 1996. Le développement : histoire d'une croyance occidentale, Paris, Presses de la Fondation nationale des sciences politiques, $426 \mathrm{p}$.

SAHLins Marshal, 2000. Identités et modernités du Pacifique, La Nouvelle revue du Pacifique 1, 1, pp. 19-25.

—, 1976. Âge de pierre, âge d'abondance. L'économie des sociétés primitives, Paris, Gallimard.

SAura Bruno, 1996. Langue, représentation du temps -mua/muri- et vision du monde à Tahiti, Bulletin de la Société des études océaniennes 269-270, XXIII, 5 , pp. 18-49.

TCHERKÉzOFF Serge., 1998. Mua/Muri : ordre, espace, et temps en Polynésie, Bulletin de la Société des études océaniennes 276, pp. 27-53.

THAYER J.S., 1983. Nature, culture and the supernatural among the Susu, American ethnologist 10, 1, pp. 116-132.

Wisner A., 1997. Anthropotechnologie. Vers un monde industriel pluricentrique, Toulouse, Octarès. 